\title{
TWO THEOREMS ON BOUNDARY VALUES OF ANALYTIC FUNCTIONS
}

\author{
S. R. BARKER ${ }^{1}$
}

\begin{abstract}
We give a new admissible maximal inequality for analytic functions of several complex variables, and also show that an analytic function which is admissibly bounded at almost all boundary points of a domain $D$ must have an admissible limit a.e. These results are then applied to the boundary behaviour of the Nevanlinna class of $D$.
\end{abstract}

We shall be concerned with the boundary behaviour of analytic functions in a smooth bounded domain in $\mathbf{C}^{n}$. For background and motivation, the reader is referred to Chapter 1 of the book by Stein [3]. The aim of this paper is to present two new results, as a corollary of which we shall be able to give very quick and simple proofs of the results in [3, Chapter 2].

The author would very much like to express thanks to his adviser Dr. D. A. Edwards for encouragement, to Professor E. M. Stein for pointing out some ambiguity in the proof of Theorem 2 in the original manuscript, and to Mrs. V. Shires for performing the typing.

Recall the definition [3, p. 32] of the admissible approach regions $Q_{\alpha}(\xi)$ at $\xi \in \partial D:$

$\mathbb{Q}_{\alpha}(\xi)=\left\{\mathbf{Z} \in D:\left|(\mathbf{Z}-\xi) \bar{v}_{\xi}\right|<(1+\alpha) \delta_{\xi}(\mathbf{Z}),|\mathbf{Z}-\xi|^{2}<\alpha \delta_{\xi}(\mathbf{Z})\right\}$,

$\delta_{\xi}(\mathbf{Z})=\min ($ dist of $\mathbf{Z}$ from $\partial D$, dist of $\mathbf{Z}$ from tangent plane at $\xi$ ),

$v_{\xi}=$ outward drawn normal at $\xi$.

The regions $\mathbb{Q}_{\alpha}(\xi)$ have conical sections in the direction of the "classical tangent" $\left\{i v_{\xi}\right\}$, but "tangential parabolic" sections in the $2 n-2$ complementary real directions (which are $n-1$ complex directions, of course).

Recall also the two types of ball on $\partial D[3$, p. 33].

The balls $B_{1}$ are the usual ones of radius $\rho$ centered at $\xi \in \partial D$, and the balls $B_{2}$ are of radius $\sim \rho$ in the classical direction, but of radius $\sim \rho^{1 / 2}$ in the complex directions. We consider also the corresponding maximal functions, denoted $_{1}^{*}$ and ${ }_{2}^{*}$.

THEOREM 1. Let $u$ be nonnegative, continuous, and plurisubharmonic in $D$ (we do not require $u$ to be continuous in $\bar{D})$, and suppose $u$ has a harmonic majorant, i.e. there is a finite positive measure $\mu$ on $\partial D$ so that $u(Z) \leqslant$

Received by the editors April 11, 1977 and, in revised furm, June 8, 1977.

AMS (MOS) subject classifications (1970). Primary 31C10, 32F05; Secondary 32A10.

Key words and phrases. Maximal function, plurisubharmonic function, almost everywhere convergence.

'Supported by the Science Research Council. 
$\int_{\partial D} P(\mathrm{Z}, W) \mu(d W)$, where $P(\cdot, \cdot)$ is the Poisson kernel $[3, p .2]$. Then the admissible maximal function of $u$, defined by

$$
M_{\alpha}(u)(\xi)=\sup _{\mathbf{Z} \in \mathbb{Q}_{\alpha}(\xi)}|u(\mathbf{Z})|
$$

satisfies

$$
M_{\alpha}(u)(\xi) \leqslant C_{\alpha}\left[\left(\left[\mu_{1}^{*}\right]^{1 / 2}\right)_{2}^{*}\right]^{2},
$$

and hence is finite almost everywhere in $\partial D$.

The point is that the right-hand expression is hardly any more complex than that in Stein's first maximal theorem [3, p. 33], and yet is easily handled in situations where the first maximal theorem could not cope (see Theorem 3 below). Stein circumvented this difficulty by using a very complicated second maximal theorem $[3$, p. 40], which we thus see may be dispensed with.

THEOREM 2. Let $F$ be holomorphic in D. If $F$ is admissibly bounded at almost all points of $\partial D$, then $F$ has an admissible limit at almost all points of $\partial D$.

Theorem 2 was only known previously in the case of a strictly pseudoconvex domain, when it was shown by an indirect argument in [3, p. 60]. As an immediate corollary we have

THEOREM 3 (STEIN). Let $F$ be holomorphic in $D$, and suppose $F$ is in the Nevanlinna class (that is, $\log ^{+}|F|$ has a harmonic majorant). Then $F$ has an admissible limit at almost all points of $\partial D$.

Proof of Theorem 1. We adopt the technique in [3, p. 33] with this crucial difference: rather than merely using the sub-mean-value property of subharmonic functions, we use the next lemma:

Lemma. Let $V$ be subharmonic and continuous on a ball $B$ in $R^{2}$ centred at the origin. Then

$$
|V(0)|^{1 / 2} \leqslant \frac{C}{|B|} \int_{B}|V|^{1 / 2}
$$

for some absolute constant $C$.

This lemma was implicit in a paper of Hardy and Littlewood [2], though it was first made explicit in [1, p. 172]. (Actually harmonicity is specified there, but a glance at the proof shows subharmonicity is all we need.)

We use the notation of $[3$, p. 36], that is, we assume the boundary point is the origin, and $v_{0}$ is in the negative $y_{1}$ direction.

Repeated application of the lemma shows that for $\mathbf{Z} \in \mathbb{G}_{\alpha}(0)$,

$$
|u(\mathbf{Z})|^{1 / 2} \leqslant \frac{C}{\rho^{n+1}} \int_{P}|u|^{1 / 2}
$$

where $P$ is the polydisc $\left\{\left|\mathbf{Z}_{1}-\xi_{1}\right|<C \rho .\left|\mathbf{Z}_{i}-\xi_{i}\right|<C \rho^{1 / 2}, i=2, \ldots n\right\}$ $\left(\rho=y_{1}\right)$. But $u(W) \leqslant C \mu_{1}^{*}(\hat{W})$ by the standard maximal theorem, where $\hat{W}$ 
is the projection of $W$ down $v_{0}$ to the boundary. So

$$
\begin{aligned}
|u(Z)|^{1 / 2} & \leqslant \frac{C_{\alpha}}{\rho^{n}} \int_{B_{2}(0, K \rho)}\left(\mu_{1}^{*}\right)^{1 / 2} d \sigma \quad \text { for } Z \in \mathbb{Q}_{\alpha}(0) \\
& \leqslant C_{\alpha}\left(\left(\mu_{1}^{*}\right)^{1 / 2}\right)_{2}^{*}
\end{aligned}
$$

and Theorem 1 is proved if $I$ can show this is finite a.e.

Recall that a function $\phi \geqslant 0$ is of weak-type $L^{p}(1 \leqslant p<\infty)$ if $|\phi>\alpha| \leqslant$ $C \alpha^{-p}$ for all $\alpha>0$, and recall also that the maximal operator

(i) maps finite measures to weak-type $L^{1}$ functions,

(ii) maps weak-type $L^{2}$ functions to weak-type $L^{2}$ functions.

(i) is standard. (ii) may be deduced from (i) either by a simple calculation, or by interpolating (i) with the trivial $L^{\infty}$ estimate for the maximal function using the interpolation theorem in [4, p. 197]. (Note that the space $L(2, \infty)$ in [4] is exactly the space of weak-type $L^{2}$ functions.) So then, $\mu$ a finite measure $\Rightarrow \mu_{1}^{*}$ is of weak-type $L^{1}$ by (i) $\Rightarrow\left(\mu_{1}^{*}\right)^{1 / 2}$ is of weak-type $L^{2} \Rightarrow$ $\left(\left(\mu_{1}^{*}\right)^{1 / 2}\right)_{2}^{*}$ is of weak-type $L^{2}$ by (ii), and, in particular, it is finite a.e.

Let us postpone proving Theorem 2 for a moment.

Proof of Theorem 3. Apply Theorem 1 to $u=\log ^{+}|F|$. We deduce $u$ and, hence, $F$ is admissibly bounded a.e. Hence by Theorem 2, $F$ has an admissible limit a.e. Q.E.D.

Proof of Theorem 2. Clearly, if $F$ is admissibly bounded a.e., $F$ is nontangentially bounded a.e. and, therefore, has a nontangential limit a.e. We now use pluriharmonicity and admissible boundedness of $F$ as Tauberian conditions. Call the nontangential limit $F_{1}(\xi)(\xi \in \partial D)$. Let $\delta>0$. We can remove an exceptional set $E(\delta)$ from $\partial D$ so that

(a) $|E(\delta)|<\delta(|E(\delta)|=$ measure of $E(\delta)$ in $\partial D)$.

(b) When $\xi \in(E(\delta))^{c}$, and $\mathbf{Z} \in \Gamma_{\beta}(\xi)$ (the nontangential approach region at $\xi$ ), then $F(\mathbf{Z})-F_{1}(\xi)$ tends to 0 uniformly in both $\mathbf{Z}$ and $\xi$ as $\operatorname{dist}(\mathbf{Z}, \xi) \rightarrow 0$.

(c) $\left|F_{1}(\xi)\right| \leqslant N(\delta)<\infty$ for $\xi \in(E(\delta))^{c}\left((E(\delta))^{c}\right.$ is the complement of $E(\delta))$.

The exceptional set $E(\delta)$ is constructed by straightforward amendment of the argument used in showing that pointwise convergence of functions is uniform outside a set of arbitrarily small measure. For each $i=2,3$, 4, etc., choose $V_{i} \subseteq \partial D$ and positive reals $K_{i}(\delta)$ so that

(i) $\left|V_{i}\right|<4^{-i} \delta$;

(ii) $\xi \in V_{i}^{c}, \mathbf{Z} \in \Gamma_{\beta}(\xi), \operatorname{dist}(\mathbf{Z}, \xi)<K_{i}(\delta)$ imply $\left|F(\mathbf{Z})-F_{1}(\xi)\right|<2^{-i}$. Also choose $V_{1} \subseteq \partial D$ so that $\left|V_{1}\right|<\delta / 2$ and $\left|F_{1}\right|$ is bounded in $V_{1}^{c}$. Then take $E(\delta)=\cup_{1}^{\infty} V_{i}$. Let $G=F_{1} \chi_{(E(\delta))}$, so $G$ is bounded and measurable. I claim $F$ has the admissible limit $G\left(\xi_{0}\right)$ at $\xi_{0} \in \partial D$, whenever $\xi_{0}$ is such that

(d) it is in the Lebesgue set of $G$ relative to the ${ }_{2}^{*}$ balls,

(e) $F$ is bounded in each admissible approach region at $\xi_{0}$,

(f) $\xi_{0}$ is a point of density of $(E(\delta))^{c}$ (relative to the ${ }_{2}^{*}$ balls).

The set of boundary points which do not satisfy these conditions is clearly of measure $<\delta$. 
For $\mathbf{Z} \in \mathbb{Q}_{\alpha}\left(\xi_{0}\right)$, let $P(\mathbf{Z})$ be the polydisc centred at $\mathbf{Z}$, whose radii are $c \delta_{\xi_{0}}(\mathbf{Z})$ in the $v_{\xi_{0}}$ direction, and $c\left(\delta_{\xi_{0}}(\mathbf{Z})\right)^{1 / 2}$ in the complex directions, where $v_{\xi_{0}}$ is the outward normal at $\xi_{0}, \delta_{\xi_{0}}(Z)$ is as in the introduction, and $c$ is a small constant. $P(\mathbf{Z}) \subseteq \mathbb{Q}_{\alpha^{\prime}}\left(\xi_{0}\right)$ where $\alpha^{\prime}>\alpha$ is independent of $\mathbf{Z}$.

Pluriharmonicity of $F$ gives $\left|F(\mathbf{Z})-G\left(\xi_{0}\right)\right|$ is plurisubharmonic and so

$$
\left|F(\mathbf{Z})-G\left(\xi_{0}\right)\right| \leqslant \frac{C}{\left(\delta_{\xi_{0}}(\mathbf{Z})\right)^{n+1}} \int_{P(\mathbf{Z})}\left|F(W)-G\left(\xi_{0}\right)\right| d \tau(W) .
$$

Let $\hat{W}$ be the projection of $W$ down $v_{\xi_{0}}$ to the boundary. Let $P_{1}(\mathbf{Z})$ be that part of $P(\mathrm{Z})$ which projects into $E(\delta)$, and $P_{2}(\mathrm{Z})$ the remainder. Split up the expression on the right:

$$
\left|F(\mathbf{Z})-G\left(\xi_{0}\right)\right| \leqslant \frac{C}{\left(\delta_{\xi_{0}}(\mathbf{Z})\right)^{n+1}} \int_{P_{1}(\mathbf{Z})}+\frac{C}{\left(\delta_{\xi_{0}}(\mathbf{Z})\right)^{n+1}} \int_{P_{2}(\mathbf{Z})}=\mathrm{I}+\mathrm{II} .
$$

Now

$$
\mathrm{I} \leqslant K \text { volume }\left(P_{1}(\mathbf{Z})\right) /\left(\delta_{\xi_{0}}(\mathbf{Z})\right)^{n+1},
$$

since $F$ is bounded in $\mathbb{Q}_{\alpha^{\prime}}\left(\xi_{0}\right)$ by (e). Hence I tends to zero as $\mathbf{Z} \rightarrow \xi_{0}$ through $\mathbb{Q}_{\alpha}\left(\xi_{0}\right)$ because of (f) above.

$$
\begin{aligned}
\text { II } \leqslant & \frac{C}{\left(\delta_{\xi_{0}}(\mathbf{Z})\right)^{n+1}} \int_{P_{2}(\mathbf{Z})}|F(W)-G(\hat{W})| \\
& +\frac{C}{\left(\delta_{\xi_{0}}(\mathbf{Z})\right)^{n+1}} \int_{P_{2}(\mathbf{Z})}\left|G(\hat{W})-G\left(\xi_{0}\right)\right|=\text { III + IV. }
\end{aligned}
$$

III tends to zero as $\mathbf{Z} \rightarrow \xi_{0}$ through $\mathcal{Q}_{\alpha}\left(\xi_{0}\right)$ because of (b).

$$
\mathrm{IV} \leqslant \frac{C}{\left(\delta_{\xi_{0}}(\mathbf{Z})\right)^{n}} \int_{B_{2}\left(\xi_{0}, K \delta_{\xi_{0}}(\mathbf{Z})\right)}\left|G(q)-G\left(\xi_{0}\right)\right| d \sigma(q),
$$

which tends to zero because of (d).

So $F(\mathbf{Z}) \rightarrow G\left(\xi_{0}\right)$ as $\mathbf{Z}$ approaches $\xi_{0}$ admissibly. Thus $F$ has an admissible limit save on a set of measure $\langle\delta$ for arbitrary $\delta>0$. Q.E.D.

It is perhaps worth remarking that by only very minor alterations to the proof above we can show:

If $F$ is holomorphic in $D$, then $F$ has an admissible limit at almost all points where it is admissibly bounded.

Again, this was only known previously when $D$ is strictly pseudoconvex.

\section{REFERENCES}

1. C. Fefferman and E. M. Stein, $H^{p}$ spaces of several variables, Acta Math. 129 (1972), 137-193.

2. G. H. Hardy and J. E. Littlewood, Some properties of conjugate functions, J. Reine Angew. Math. 167 (1932), 405-423. 
3. E. M. Stein, Boundary behaviour of holomorphic functions of several complex variables, Princeton Univ. Press, Princeton, N. J., 1972.

4. E. M. Stein and G. Weiss, Introduction to Fourier analysis on Euclidean spaces, Princeton Univ. Press, Princetoi, N. J.,1971.

MAthematical InstituTe, 24-29 St. Gnes, OxPORD, UNTted KnNGdOM

Current address: Wolfson College, Oxford, United Kingdom 